\title{
Study of hygrothermal ageing of glass fibre reinforced PET composites
}

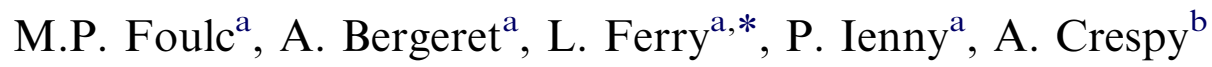

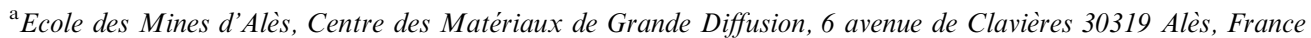 \\ ${ }^{\mathrm{b}}$ Université de Toulon et du Var, Bâtiment R 118, BP 132, 83957 La Garde Cedex, France
}

\begin{abstract}
Hygrothermal ageing has been investigated on glass fibre reinforced polyethylene terephthalate (PET) composites using complementary techniques and a multiscale approach in order to identify the different steps of the material's degradation. For early ageing times $(t<6 \mathrm{~h})$, DMTA tests give evidence of the plasticisation of the PET matrix. GPC measurements and acid end group titration show that the chemical degradation step of the composites occurs immediately and that the main degradation mechanism is random chain scission. The changes in morphology resulting from hydrolysis, investigated through DSC and SAXS experiments, reveal a decrease in the long period that may result from the diffusion of oligomers out of the spherulites. The water uptake for long ageing times is attributed to an interfacial debonding which induces an osmotic pressure in this area. Photomechanical measurements highlight the development of microcracks within the aged material that induced an increase in the variation of material volume.
\end{abstract}

Keywords: PET composites; Hygrothermal ageing; Plasticisation; Hydrolysis; Osmotic cracking; Interfacial debonding; Photomechanics

\section{Introduction}

Thermoplastic composites are more and more used in applications in which they are exposed to severe environments combining temperature and moisture. In the literature, many studies are dedicated to the hygrothermal ageing of various polymer matrices and their composites: high temperature engineering polymers $[1,2]$, polyamides [3-5], polyesters [6-8].

PET is a semi-crystalline polyester that confers stiffness, temperature performance, dimensional stability and high gloss finish to glass reinforced composites. The inherent hydrolytic fragility of the ester link justifies

\footnotetext{
* Corresponding author. Tel.: +3346678 53 58; fax: +3346678 5365 .

E-mail address: laurent.ferry@ema.fr (L. Ferry).
}

the interest of scientists in PET ageing in water or humid atmospheres [9-14].

Water absorption induces a severe degradation of the mechanical properties of the composites that are not only related to physical, i.e. plasticisation, and chemical, i.e. hydrolysis, modification of the polymer matrix but also to debonding affecting the fibre-matrix interface. Plasticisation is supposed to be a reversible phenomenon [15] while chemical ageing, occurring for long ageing times, irreversibly affects the molecular structure inducing chain scissions and thus embrittlement [16]. Interfacial debonding induces osmotic cracking damage, typically obtained in the case of unsaturated polyester resins for marine applications $[17,18]$.

Up to now, the hygrothermal ageing of composites was investigated under two different approaches. On one hand, chemists aim at quantifying chemical degradation of the matrix. Lifetime prediction models are more or 
less simple depending on the phenomena taken into account. The simplest models derive directly from the basic second order kinetic equation in which the water concentration is considered constant [9] or conversion dependant [14]. Some authors assume the existence of autocatalysis [10,11], others consider the case of diffusion controlled kinetics [12]. On the other hand, the mechanical approach aims at modelling the evolution of the mechanical properties during ageing [19-23].

The purpose of this paper is to propose a global approach to study the hygrothermal ageing of PET composites which is based on a multiscale analysis (macro-, meso- and micro-scale analysis) of the different steps of the damage process. A first part of the study will be focused on the evaluation of the decrease in the macroscopic properties, such as mechanical properties, with ageing time. Then a second part of this study will concern the identification of the chemical reactions occurring during ageing at a microscopic scale. Finally a third part will study the changes of the microstructure and the occurrence of interfacial debonding generated by hydrolysis thanks to a mesoscopic approach carried out through photomechanical measurements.

\section{Experimental}

\subsection{Material and ageing conditions}

Short glass fibre reinforced PET composites were supplied by DuPont de Nemours Company (Geneva, Switzerland) under the trademark Rynite ${ }^{\circledR}$. The selected grade for this study is $30 \%$ by weight glass fibre reinforced. After the injection moulding step, the samples (ISO 527-2 type 1-A) were aged by immersion in deionised water in an autoclave at $120{ }^{\circ} \mathrm{C}$ (1.6 bars).

\subsection{Measurement of water absorption}

Water uptake was first measured by weighing the samples periodically at ambient temperature. The relative weight gain of the matrix was plotted versus the square root of time. The water content was also measured by Karl Fisher titration which consists in a potentiometric titration (Metrohm Titrino) based on a redox reaction between iodine molecules and water molecules. Water molecules were desorbed at $200{ }^{\circ} \mathrm{C}$ under nitrogen and then carried by the gas flow to the titration cell.

\subsection{Mechanical characterization}

Maximum tensile strength was measured according to the ISO 178 standard (speed: $5 \mathrm{~mm} / \mathrm{min}$ ) using an Adamel Lhomargy universal testing machine. Unnotched Charpy impact strength was determined using a Zwick apparatus according the ISO 179 standard.
The photomechanical technique is an optical method which allows the evaluation of the in-plane kinematic surface field without contact. This technique was adapted from a uniaxial tensile test (Dartec M1000/RE $100 \mathrm{kN})$. The digital image correlation software (CinE$\mathrm{MA}^{(}$) allows the quantification of the components of the Green Lagrange strain tensor at any point of the surface of the tested sample. The experimental set-up is composed of a white-light source, a high resolution CCD camera (KODAK Megaplus 1.4, $1024 \times 1024$ squared and jointed pixels) kept in front of the specimen. The principle of correlation consists in the observation of a geometrical pattern on the sample surface before and after the displacement during the tensile test. The image processing is based on a coarsefine search method leading to the two-dimensional displacement components with a subpixel accuracy. Applied to all the points that constitute the initial mesh, this treatment provides strain fields. Hereafter the digital image processing is described for a pair of successive images. This procedure is applied to the whole set of images recorded during the mechanical test. At the first step of digital image processing, a virtual meshing is defined on the reference image. The user has to specify the number of points for both axial and transverse directions which define the corresponding distance (in pixels) between two points (GS as grid step). Each point of the virtual mesh corresponds in fact to the centre of the so-called pattern. This pattern is defined by its correlation size (CS). It is the elementary cell representative of the material point which has been tracked. The maximum displacement value $\left(V_{\mathrm{m}}\right)$ in the axial and the transverse directions has to be finally specified. A first correlation calculation, performed on this predefined mesh, gives access to the rigid body displacement components. A subsequent reference change is performed on the second image, relative to the first one, in such a way that the displacement field computed at the next step is the only deformation-process-induced one. The second step of pair-of-images-processing consists in evaluating the two-dimensional field of in-plane displacements. Once again, the user has to specify the correlation parameters (CS, GS and $V_{\mathrm{m}}$ ) for a virtual mesh corresponding to a given number of digital extensometers. According to the two-dimensional coordinate system, gradient tensor and finally components of the in-plane strain tensor are evaluated.

\subsection{Physical characterization}

The dynamic mechanical properties were studied with a VA 815 Metravib RDS apparatus at a heating rate of $5{ }^{\circ} \mathrm{C} / \mathrm{min}$ over a temperature range from -150 to $230{ }^{\circ} \mathrm{C}$. The samples $\left(60 \times 10 \times 4 \mathrm{~mm}^{3}\right)$ were tested using an imposed frequency of $10 \mathrm{~Hz}$ and an oscillation amplitude of $10 \mu \mathrm{m}$ in the bending mode. The purpose 
of this analysis consisted in the determination of the main relaxation characteristics for different ageing times.

DSC experiments were carried out with a SETARAM DSC 92 instrument at a $10{ }^{\circ} \mathrm{C} / \mathrm{min}$ heating rate under nitrogen atmosphere to study the influence of the ageing on the melting temperatures. Thermogravimetry (TGA) measurements were performed on a SETARAM TGDTA 92 apparatus at a heating rate a $10{ }^{\circ} \mathrm{C} / \mathrm{min}$ under nitrogen atmosphere to identify the nature of the PET oligomers present in the solubilised fraction.

ATR infrared spectra were recorded at room temperature on a Bruker IFS66 FTIR spectrometer (Golden Gate reflection system) to analyse the solubilised fraction of the aged PET composites. Each spectrum is an average of 64 spectra measured at a resolution of $2 \mathrm{~cm}^{-1}$.

SAXS experiments were performed on parallelepiped-like samples (1 mm thick), on the D2AM synchrotron beamline of ESRF (Grenoble, France). The collected scattered intensities were corrected taking into account the background scattering. The monodimensional intensity function $q^{2} I(q)$ was plotted versus the scattering vector $q$. The value of $q$ corresponding to the first maximum of this function allows the calculation of the long period thanks to the Bragg's law:

$\lambda=2 L \sin \theta_{\mathrm{B}}$

where $\lambda$ is the wavelength, $L$ the distance between scattering elements and $\theta$ the scattering angle.

\subsection{Chemical characterization}

According to Pohl's method [24], carboxyl terminal free groups were determined by specific titration using a methanolic sodium hydroxide solution (concentration: $0.1 \mathrm{moll}^{-1}$ ). The solvent system used was a mixture of benzyl alcohol and chloroform which forms a metastable solution able to solubilise the polymer as well as degraded samples containing up to $0.8 \mathrm{meq}$ carboxyl groups per $g$ of polymer. The titrations were carried out at a temperature higher than $100{ }^{\circ} \mathrm{C}$ in order to avoid the reprecipitation of the polymer.

Gel permeation chromatography analysis was performed at $100{ }^{\circ} \mathrm{C}$ on a Waters $150 \mathrm{C}$ chromatography system equipped with a differential refractometer and with three columns of crosslinked polystyrene coupled in series (Catalyse, Marseille, France). Samples were prepared by dissolving the composite in $m$-cresol at $100{ }^{\circ} \mathrm{C}$ in order to reach a final concentration of $0.5 \%$ $(w / v)$. The solutions were filtered in order to eliminate glass fibres. The calibration of the columns $(300 \times$ $7.5 \mathrm{~mm}^{2}$ ) was carried out by using polystyrene standards of known molecular weight and polydispersity. $m$-Cresol was used as eluent with a flow rate of $0.5 \mathrm{ml} / \mathrm{min}$ and the injected sample volume was $250 \mu 1$.

\section{Results and discussion}

\subsection{Effect of hygrothermal ageing at a macroscopic scale}

The variations of the macroscopic mechanical properties of PET composites during the ageing test are shown in Figs. 1 and 2. A drastic decrease in the ultimate properties (Fig. 1) and in impact strength (Fig. 2) was highlighted until $45 \mathrm{~h}$ of ageing. This decrease is related to the chemical degradation of the polyester matrix which starts immediately. This evolution of impact strength with ageing time is in good agreement with those observed in a previous study on PBT composites [25]. It differs notably from the behaviour of PA-6,6 composites, where a slight increase in the impact strength can be observed during the first hours of ageing which may highlight the plasticisation of the matrix.

Another effect of ageing at the macroscopic scale is a mass uptake phenomenon. Fig. 3 shows the weight change of the sample caused by water absorption as a function of ageing time. This evolution can be divided in two parts: (i) a first part characterized by a non-linear increase in the mass uptake, and (ii) a second part after $60 \mathrm{~h}$ of ageing time where a decrease in the mass uptake was observed. This curve does not correspond to a Fickian behaviour related to a single free-phase diffusion since saturation is not observed. For the first non-linear increase, the diffusion model of Langmuir [26] seems to be the more accurate model for PET composites as the random mobility of water molecules has a low probability because of the reactivity of ester groups. This model assumes that a fraction of the water molecules is fixed to polar sites and that the other fraction is free to diffuse within the polymer matrix. It can be noticed that Adamczak [15] has observed Fickian

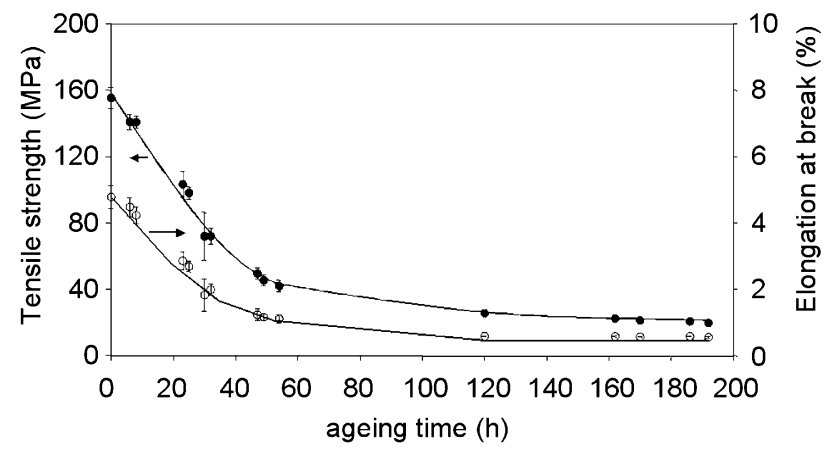

Fig. 1. Evolution of tensile strength and elongation at break with ageing time. 


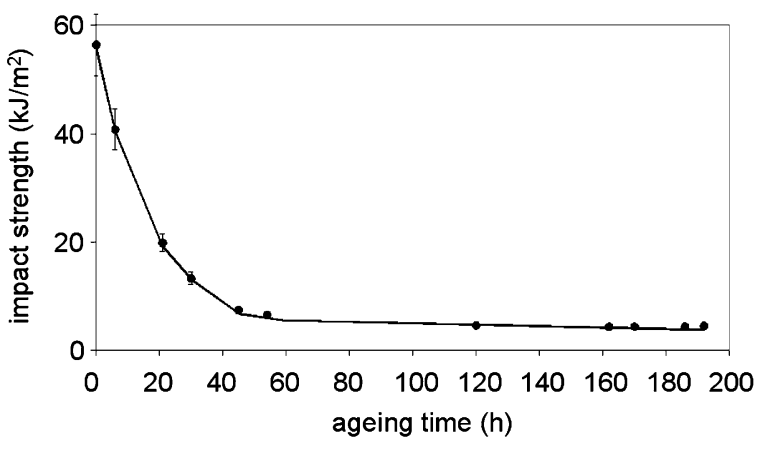

Fig. 2. Evolution of impact strength with ageing time.

behaviour for ageing temperature below $90{ }^{\circ} \mathrm{C}$ for various PET composites. The decrease in the mass uptake, that has been already observed for polyamide6,6 composites [5], may be attributed to the diffusion and extraction of solubilised oligomers. In this case the mass of extracted oligomers was assumed to be higher than the water uptake. In order to confirm this hypothesis, a quantification of the fraction of solubilised oligomers with ageing time was investigated. Fig. 3 shows that the solubilised fraction increases after about $50 \mathrm{~h}$ of ageing time. This result may explain the decrease in the mass uptake for long ageing times. Thermogravimetric analysis (TGA) experiments, FTIR and DSC characterizations were also carried out to complete this study. TGA analysis (Fig. 4) performed on the solubilised fraction collected after an ageing time of $170 \mathrm{~h}$ give evidence of a three-step degradation mechanism which remains close to those of the PET composite. The additional weight loss at about $265{ }^{\circ} \mathrm{C}$ can be related to a possible condensation reaction of oligomers during the heating in TGA experiment. Moreover, as the weight loss of the solubilised fraction is different from those of terephthalic acid, it can be concluded that the ageing does not lead to the formation of monomers even for long ageing times, even if FTIR spectra (Fig. 5) are almost similar. Nevertheless the presence of specific peaks located, on one hand at

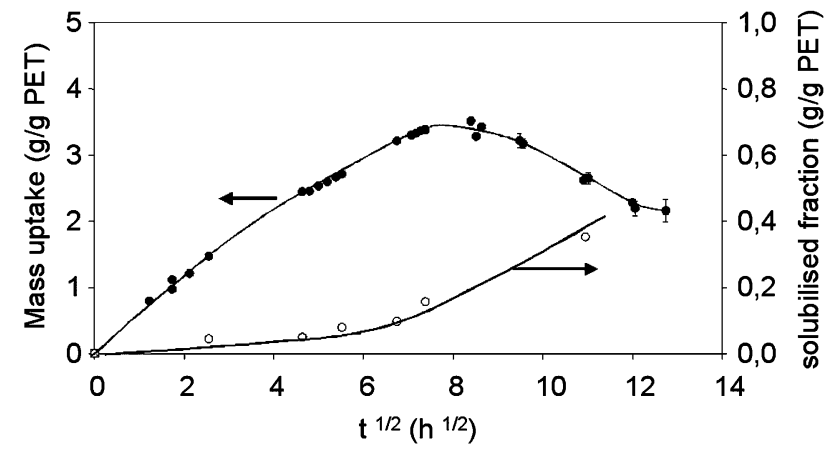

Fig. 3. Evolution of mass uptake $(\bullet)$ (gravimetric measurements) and of the solubilised fraction $(O)$ with ageing time.

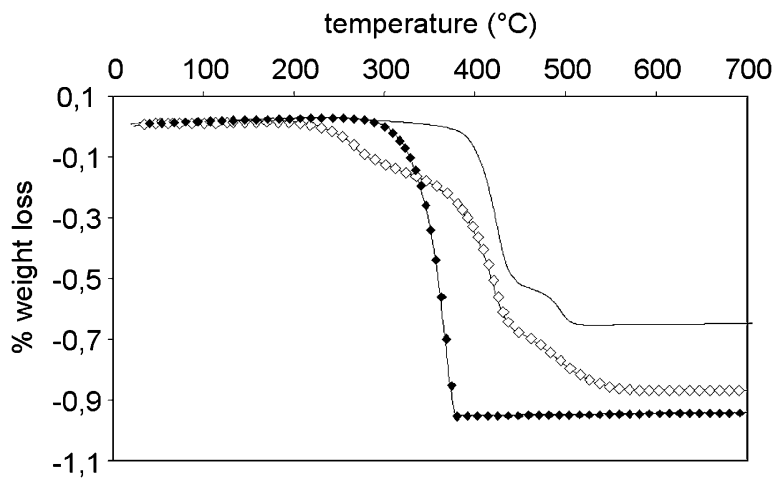

Fig. 4. Variation of the mass loss versus temperature (TGA experiments) for $(-)$ PET composite, $(\diamond)$ solubilised fraction after an ageing time of $120 \mathrm{~h},(\diamond)$ terephthalic acid.

$1405 \mathrm{~cm}^{-1}\left(\nu_{\mathrm{C}-\mathrm{OH}}\right.$ (acid) $), 1270 \mathrm{~cm}^{-1}\left(\nu_{\mathrm{C}-\mathrm{O}}\right.$ (acid) $)$ and $923 \mathrm{~cm}^{-1}\left(\nu_{\mathrm{H} \cdots \mathrm{OH}}\right.$ (acid out of plane) $)$, on the other hand at $3453 \mathrm{~cm}^{-1}\left(\nu_{\mathrm{OH}}\right.$ (alcohol dimer I) $)$ and $1068 \mathrm{~cm}^{-1}\left(\nu_{\mathrm{C}-\mathrm{C}-\mathrm{O}}\right.$ out of plane alcohol) $)$, corresponding to acid and hydroxyl end groups, give evidence that all combinations of the end groups are possible for these oligomers. As the nature of these end groups influences the melting behaviour of these oligomers, DSC experiments were performed (Fig. 6). It can be noticed that no exothermic reaction occurred during the temperature increase, so that the condensation reaction that was observed during TGA experiments is not present in this case. The solubilised fraction collected after an ageing time of $162 \mathrm{~h}$ shows a single and large melting peak located at about $220{ }^{\circ} \mathrm{C}$, that may correspond to a hydroxy-acid oligomer with $n=3$. For longer ageing times (170 and $192 \mathrm{~h})$ several DSC peaks were detected at $176^{\circ} \mathrm{C}$, $225^{\circ} \mathrm{C}$ and $245^{\circ} \mathrm{C}$, that may be related, respectively, to a hydroxy-acid oligomer with $n=1$, with $n=3$ and to the PET matrix. Therefore it can be concluded that a distribution of oligomers characterized by different chain lengths has been formed.

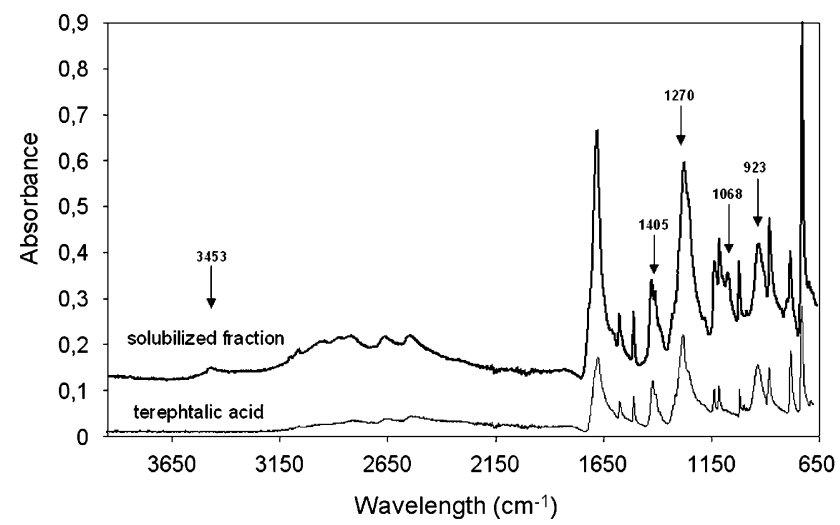

Fig. 5. FTIR spectra of solubilised fraction after an ageing time of $170 \mathrm{~h}$, terephthalic acid. 


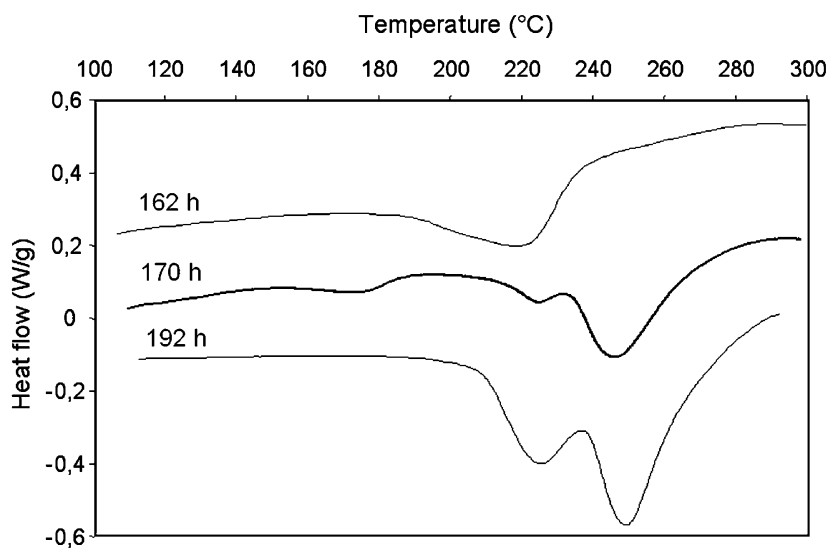

Fig. 6. DSC thermograms of solubilised fraction after $162 \mathrm{~h}, 170 \mathrm{~h}$, and $192 \mathrm{~h}$ of ageing.

In order to quantify the water content for long ageing times, Karl Fisher titrations were performed as shown in Fig. 7. A new increase in the water content can be observed for long ageing times, over $120 \mathrm{~h}$. This increase results from an interfacial debonding between the glass fibre and the matrix as confirmed by SEM micrographs (Fig. 8). This debonding induces a new water uptake because of the formation of cracks or voids in the bulk material. The existence of differential pressures between the low and the high water content regions induces an osmotic cracking phenomenon. Moreover, it can be noticed that no correlations exist between the mass uptake measured through gravimetric experiments and the water content determined through Karl Fisher titrations because of difference in sample preparation.

\subsection{Identification of the different steps of degradation}

\subsubsection{Plasticisation step}

It has been well established that the absorbed water has a plasticising effect on the matrix and causes finally a loss in the mechanical performance. In order to identify this first step of degradation, the dynamic mechanical behaviour in the $\alpha$ region of different aged

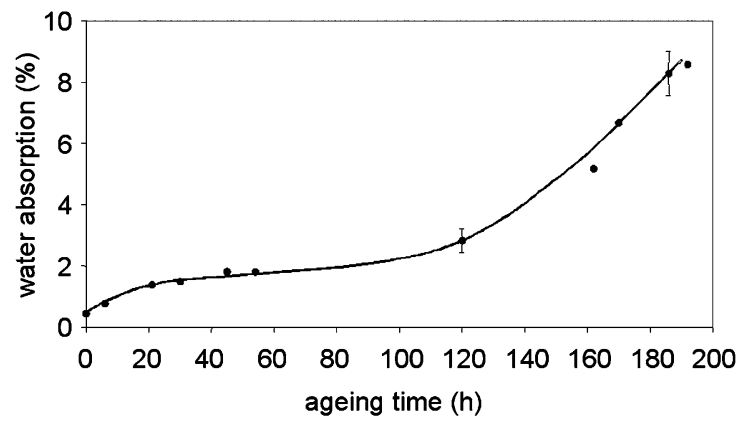

Fig. 7. Evolution of the water absorption with ageing time (Karl Fisher titrations).
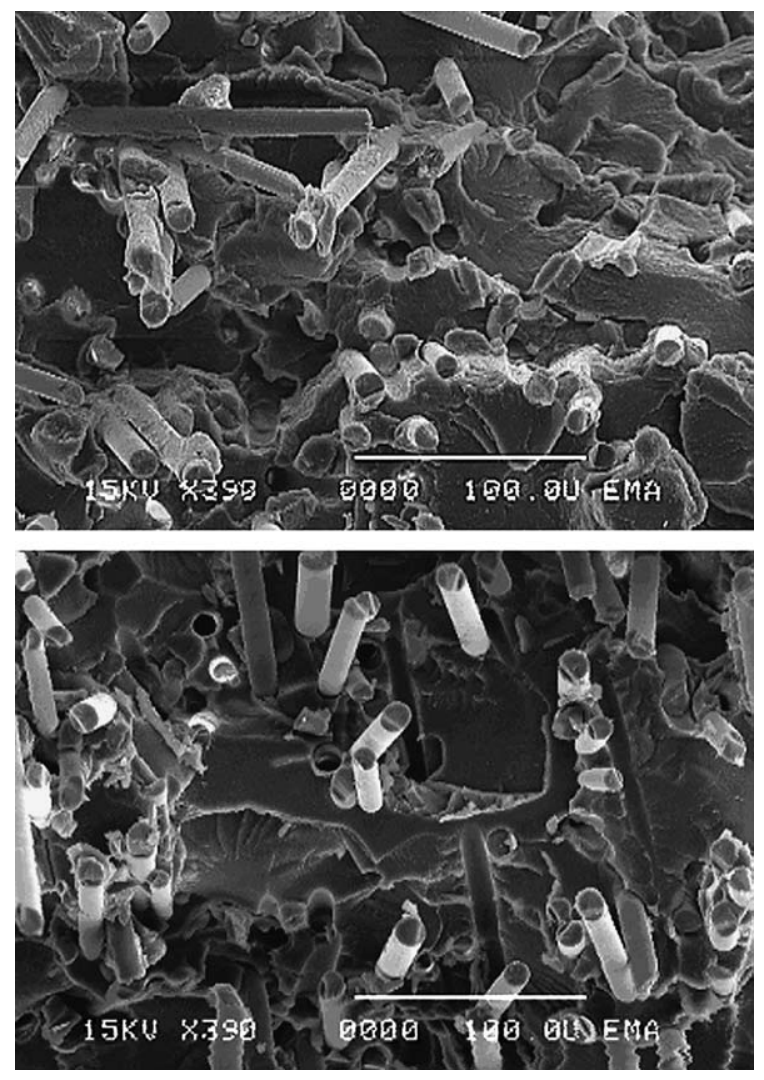

Fig. 8. SEM micrographs $(\times 390)$ of broken cross sections of PET composites for unaged composite (a) and composite aged for $54 \mathrm{~h} \mathrm{(b)}$.

composites were investigated (Fig. 9). For the unaged samples, the $\alpha$ relaxation related to the glass transition occurs at around $T_{\alpha}=104{ }^{\circ} \mathrm{C}$. A decrease of about $9{ }^{\circ} \mathrm{C}$ in the $T_{\alpha}$ temperature was observed between the unaged sample and the sample aged for $6 \mathrm{~h}$ where absorption of $1.5 \%$ of the initial mass of the matrix has been previously measured. This phenomenon is related to plasticisation, which induces an increase in the amorphous chain mobility. For ageing times over $6 \mathrm{~h}$, a decrease in the magnitude of the $\alpha$ relaxation peak has

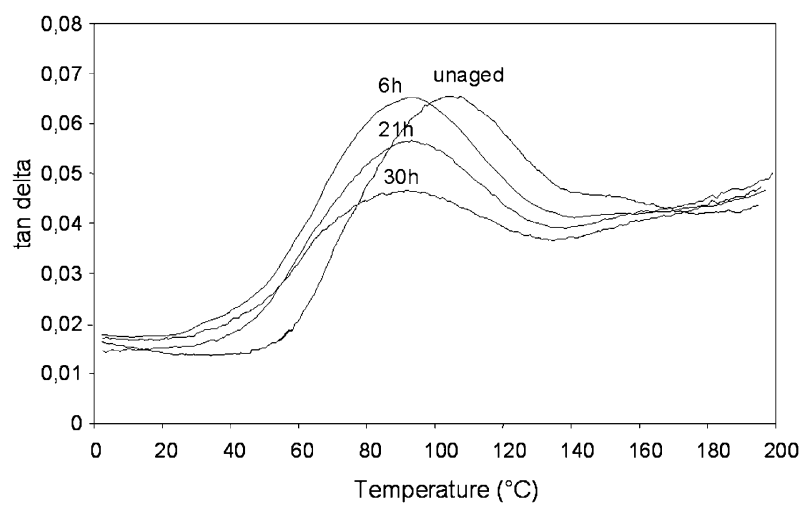

Fig. 9. Dynamic mechanical behaviour: dissipation factor $\tan \delta$ versus temperature in the $\alpha$ region after various ageing times. 
been observed and show severe changes in the molecular structure of the matrix. This decrease indicates that a lower amount of amorphous chains are able to contribute to cooperative movements. This can be due to the extraction of hydrolysed oligomers or to the crystallization of these later in small crystalline entities. Then, chemical characterizations have been carried out in order to have information about the effect of hydrolysis on the chemical structure.

\subsubsection{Chemical degradation step}

It has been generally established that the hydrolysis of most polyesters induces the production of acidic end groups and so, a decrease in the molecular weight. This hydrolysis reaction can take place through two mechanisms: (i) a random chain scission mechanism where any ester bond has the same probability to be hydrolysed [27], and (ii) a classical depolymerisation process [28]. The decrease in the molecular weight with increasing ageing time (Fig. 10) gives evidence of a twostep mechanism: (i) up to about $100 \mathrm{~h}$ of ageing, $M_{\mathrm{w}}$ seems to decrease quasi-hyperbolically with time. This result may assume the main presence of a random chain scission mechanism; (ii) beyond $120 \mathrm{~h}$ of ageing, $M_{\mathrm{w}}$ can be considered as constant and tends towards an asymptotic value of about $5400 \mathrm{~g} \mathrm{~mol}^{-1}$. This second quasi-linear part of the curve indicates the presence of a fraction non-reactive or less reactive towards water molecules. As hydrolysis mainly occurs within the amorphous phase and at chain folds [16,29], this fraction probably corresponds to crystalline structures that are impermeable to water. As a conclusion, it can be assumed that the major part of the amorphous phase has been hydrolysed before $100 \mathrm{~h}$ of ageing and that the probability to generate chain recombination is low. In order to have more information about the scission mechanism, the polydispersity index has been measured (Fig. 10). A pseudo hyperbolic decrease in polydispersity index with ageing time has been observed. The same

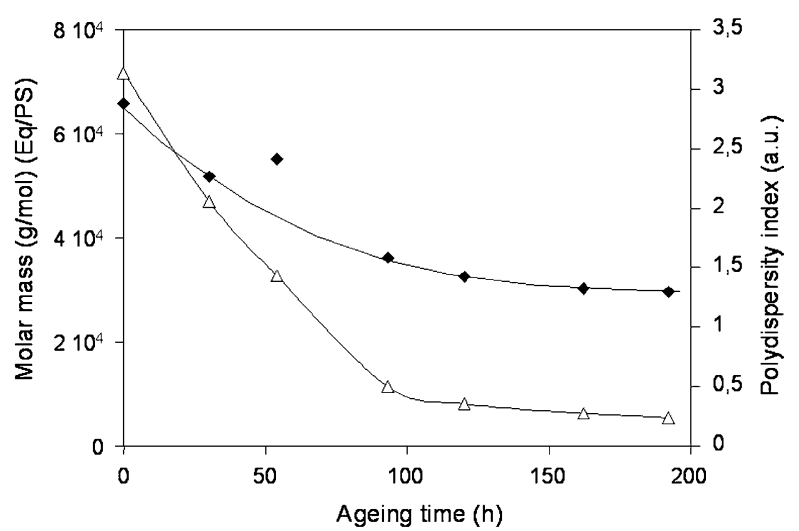

Fig. 10. Molecular mass and polydispersity index variations with ageing time. variations have been obtained by Launay et al. [16] on amorphous PET films aged in boiling water. These authors assumed that the random chain scission mechanism is predominant and that hydrolysis remains homogeneous at a dimension scale higher than the thickness of the crystalline lamella. The evolution of the number of chain scission $n_{t}$ estimated thanks to the initial molecular weight $M_{n 0}$ and that of the aged sample $M_{n t}$ ( $t$ being the ageing time) through the following relation can be determined:

$n_{t}=\frac{1}{\bar{M}_{n t}}-\frac{1}{\bar{M}_{n 0}}$

From this result (Fig. 11), the autoaccelerated character of the reaction was clearly identified up to $120 \mathrm{~h}$ of ageing. For longer ageing times, the number of chain scissions increases more slowly. This phenomenon results from the extraction of oligomers and indicates that the major part of the amorphous phase was hydrolysed at this time. Moreover, the $n_{t}$ evolution follows the variations of the number of acid end groups evolved during ageing as it can be observed in the same figure. The slow increase in the $\mathrm{COOH}$ amount observed up to $54 \mathrm{~h}$ of ageing is associated to a fast decrease in the molecular weight. This result confirms the predominance of random scission in this early stage of degradation. Indeed a depolymerisation mechanism would induce a faster increase in the rate of acidic chain end production. Another reason for this relatively moderate acidic end group formation rate is that the water diffusion may control the kinetics of hydrolysis. In this later hypothesis, the number of ester bond scissions will be lower in the core than in the skin of the sample.

Between $54 \mathrm{~h}$ and $120 \mathrm{~h}$ of ageing, the fast increase in acidic end group formation rate is connected to a still important decrease in molecular weight and may result from an homogenisation of the water content throughout the sample. The last part, corresponding to ageing times longer than $120 \mathrm{~h}$, highlights a slowdown in the

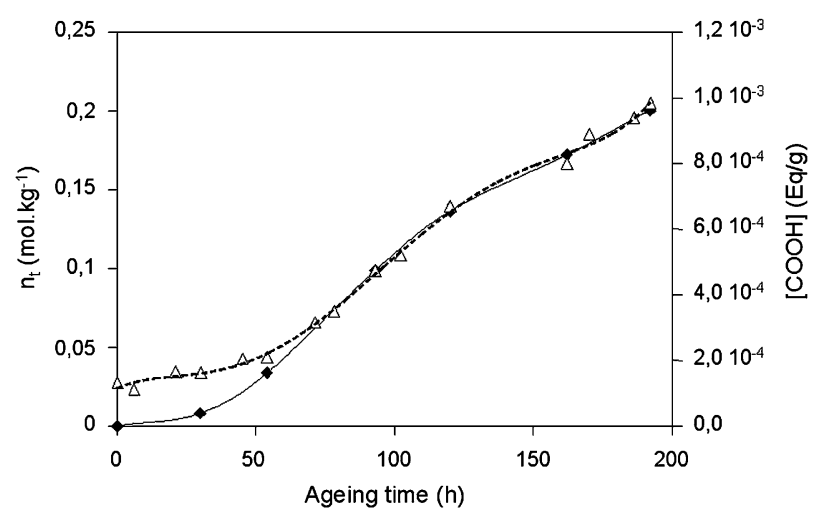

Fig. 11. Evolution of the number of chain scission $n_{t}(\bullet)$ and of the acidic end chains rate $(\Delta)$ with ageing time. 
rate of increase of $\mathrm{COOH}$ content due to the extraction of oligomers previously described.

\subsubsection{Morphological evolution}

The morphological changes induced by chain scissions during ageing have been investigated by DSC. A first increase in crystallinity (Fig. 12) from 27 to $40 \%$ is observed before $54 \mathrm{~h}$ of ageing and can be attributed to a higher crystallization ability of shorter molecular chains resulting from chain scissions due to an enhanced mobility. For longer ageing times, it is difficult to conclude because two coexistent phenomena may affect the crystallinity ratio. On one hand, the preferential removal of amorphous material during degradation results in an increase of crystallinity of the remaining material. On the other hand, for long ageing times, it can be assumed that crystalline lamellae will be also partially attacked by hydrolysis inducing a slight decrease of the crystallinity ratio.

This later hypothesis is corroborated by the evolution of the melting temperature $\left(T_{\mathrm{m}}\right)$ as a function of ageing time (Fig. 12). A significant decrease of $T_{\mathrm{m}}$ is observed beyond $70 \mathrm{~h}$ of ageing. According to the GibbsThomson relation, a decrease of $T_{\mathrm{m}}$ can be assigned either to a decrease of the lamellar thickness or to a change in amorphous-crystal surface energy, both being likely to occur simultaneously. In order to point out which of both phenomena is responsible for the evolution of $T_{\mathrm{m}}$, Small Angle X-ray Scattering (SAXS) experiments have been carried out.

The long spacing $L$, characterizing the repeating length within lamellar stacks, has been determined using the one-dimensional correlation function of the Fourier transform of SAXS profiles in real space. Fig. 13 shows that the maximum of the one-dimensional correlation function is shifted to high values of the scattering vector $q$ as ageing progresses. This result indicates that the long spacing decreases with ageing time. Two assumptions may be proposed to explain this variation. (i) The

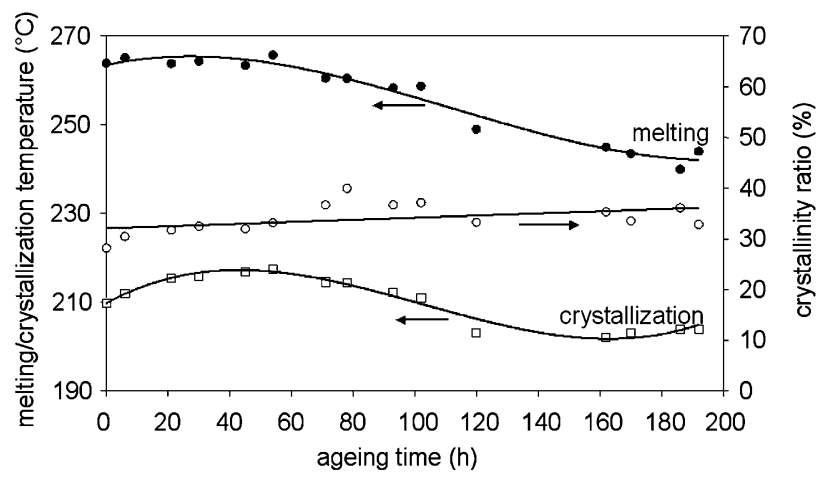

Fig. 12. Evolution of crystallinity ratio $(\bigcirc)$, melting peak temperature $(\bullet)$, and crystallization temperature $(\square)$ with ageing time.

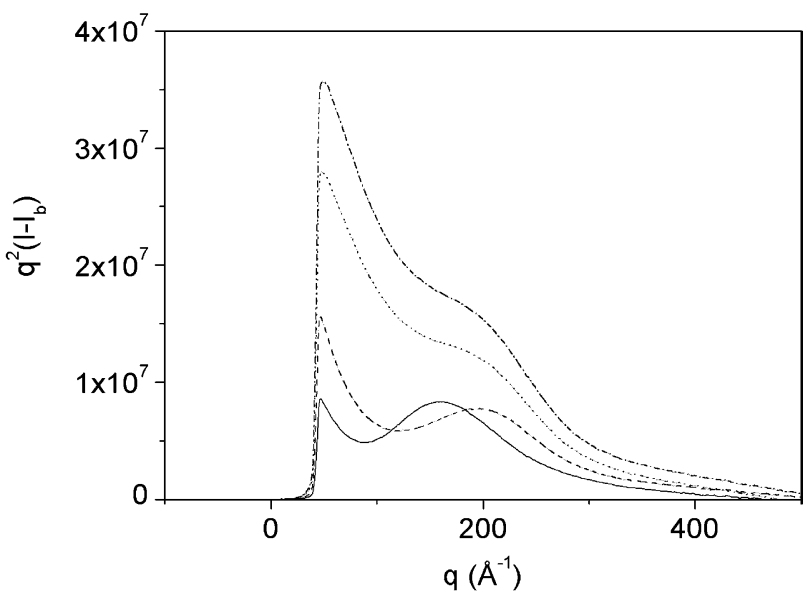

Fig. 13. Plot of monodimensional intensity function $q^{2} I(q)$ versus scattering vector $q$ for different aged samples: (-) unaged, (- - -) $162 \mathrm{~h}$ aged, (...) $186 \mathrm{~h}$ aged, and ( - . - $) 192 \mathrm{~h}$ aged.

decrease of $L$ may result from the crystallization of oligomers generated by hydrolysis. The new crystalline entities would be intercalated between the initial lamellae reducing the distance between them. This hypothesis seems not reasonable since it would decrease the long spacing by a factor 2 and implies a high increase in crystallinity not consistent with the experimental results. (ii) The decrease of $L$ may be attributed to a structural relaxation associated with chain scissions in the amorphous phase as proposed by King et al. [30]. Macromolecules in tension in the amorphous phase could relax after bond scission leading to a contraction of the structure. This phenomenon may be facilitated by the extraction of oligomers from the interlamellar zone. Such oligomers could be either evacuated in the ageing bath (see solubilised fraction) or could recrystallize in the interspherulitic zone in small crystallites without any periodic stacking. For very long ageing time, a partial hydrolysis of the crystalline phase may occur. This second interpretation seems in good agreement with the results obtained by DSC.

\subsubsection{Interfacial debonding}

Fig. 13 reveals also that the scattering intensity at very small angles increases with ageing time. Besides, this increase leads to mask the correlation peak connected to lamellar stacks. This additional scattering may be related to decohesion occurring at the polymerfibre interface. Debonding induces cavities or microcracks that can act as scatterers for the X-ray beam. This result is in good agreement with the new increase in the water content observed for long ageing times (Fig. 7). In order to confirm this last result, photomechanical characterizations were investigated.

The chemicrystallization resulting from hydrolysis is responsible for a slow down of the diffusion process for 
long ageing times. This tendency seems to be compensated by interfacial debonding that induces an important subsequent absorption of water. As mentioned above, the interface between the glass fibre and the matrix constitutes a privileged site for the water molecules accumulation during the diffusion process. It has been proved that these water molecules were able to hydrolyse the curing agents at the interface, then inducing debonding and the formation of microcracks for long ageing times. Once the water molecules have diffused in these voids, the solubilisation of organic molecules is responsible for the development of an osmotic pressure inside the microcracks. The increase of this pressure induces a crack at the sample surface and corresponds to the ultimate step of the hydrolysis damage process [17].

The debonding at the interface induces modifications of the local strain behaviour of the sample. Thus, photomechanical experiments were performed in order to characterize the in-plane displacements at the sample surface during a tensile test. Two meshes were defined (Table 1): the rough one is devoted to the study of the macroscopic behaviour, and the thin one, enables us to quantify the in-plane local strains. The studied composite being characterized by a viscoelastic behaviour, a relaxation step has been performed between the two sequential loading phases. Therefore elastic modulus is interpreted from the unloading phase to occult the material viscosity. The following loading step is conducted until the fracture of the specimen. For both last sequences, the in-plane strain tensor has been measured by using photomechanical technique described previously.

3.2.4.1. Study of the macroscopic behaviour. For the unloading stage, the linear evolution of the applied stress versus the macroscopic longitudinal strain enables to quantify the Young's modulus of the different aged composites. A very fast decrease in the pure elastic Young's modulus resulting from the hydrolysis of the matrix is observed till $120 \mathrm{~h}$ of ageing. Beyond this time, no clear tendency can be observed. Moreover, the disappearance of the transverse strain expressed by a decrease in the Poisson's ratio was highlighted too (Fig. 14). This later gives evidence that interfacial debonding is initiated from the first hours of ageing and becomes significant between $60 \mathrm{~h}$ and $120 \mathrm{~h}$. Photomechanics allowed also to study the volume variation of

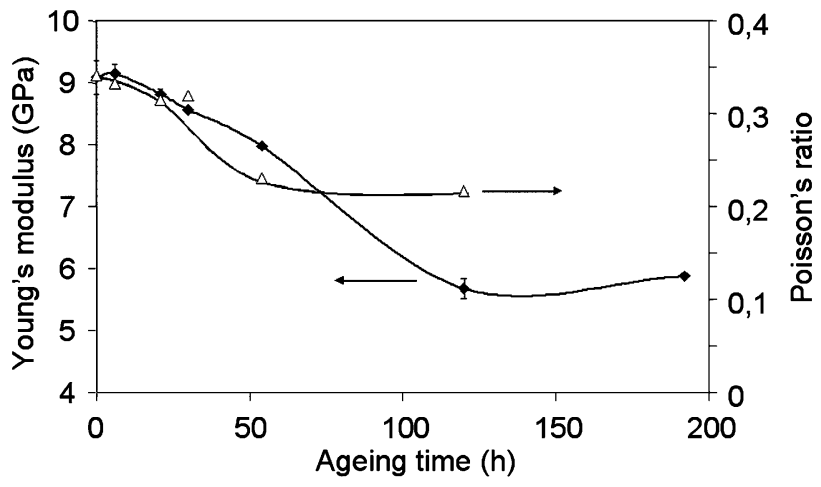

Fig. 14. Evolution of the elastic properties during ageing: Young's modulus $(\diamond)$ and Poisson's ratio $(\triangle)$.

the selected area during the tensile test. Assuming transverse isotropy of the material (Eq. (3)) and the small strain hypothesis (Eq. (4)) the volume variation parameter is calculated from the trace of the Lagrange's strain tensor:

$L_{x x}=L_{z z}$

$\left(\frac{\Delta V}{V_{0}}\right)_{\exp }=\sum \varepsilon_{i i} \approx \sum L_{i i}=2 L_{x x}+L_{y y}$

If it is supposed that the response of the material is elastic, a theoretical relation between the volume variation and the applied stress and strain can be given as the following (Eq. (5)):

$\left(\frac{\Delta V}{V_{0}}\right)_{\text {theo }}=(1-2 \nu) L_{y y}=\left(\frac{1-2 \nu}{E}\right) \sigma_{y y}$

For different aged samples, the evolution of the theoretical (Eq. (5)) and experimental (Eq. (4)) volume variations is represented in Fig. 15. The deviations between experimental and theoretical curves should suggest the formation of cracks and cavities within the sample. It was observed that the unaged sample and the sample aged for $6 \mathrm{~h}$ present an elastic volume variation until a high longitudinal strain $L_{y y}$ of about $5 \times 10^{-2}$. Beyond this value of strain, the experimental volume variation becomes higher than the analytical one. This may probably due to the formation of cracks or cavities inside the sample. For aged samples, the deviation from

Table 1

Parameters of correlation employed for the photomechanics technique

\begin{tabular}{lll}
\hline & Macroscopic behaviour & Local behaviour \\
\hline Selected area & $249 \times 249$ pixels $(7.5 \times 7.5 \mathrm{~mm})$ & $483 \times 279$ pixels $(16.1 \times 9.3 \mathrm{~mm})$ \\
Grid step (Gs) & 31 pixels $(0.93 \mathrm{~mm})$ & 3 pixels $(1 / 10 \mathrm{~mm})$ \\
Total number of points $(\mathrm{Ns})$ & $9 \times 9(81$ points $)$ & $162 \times 94(15228$ points $)$ \\
Extensometric gauge $(\mathrm{Lg})$ & 248 pixels $(7.4 \mathrm{~mm})$ & 18 pixels $(6 / 10 \mathrm{~mm})$ \\
\hline
\end{tabular}




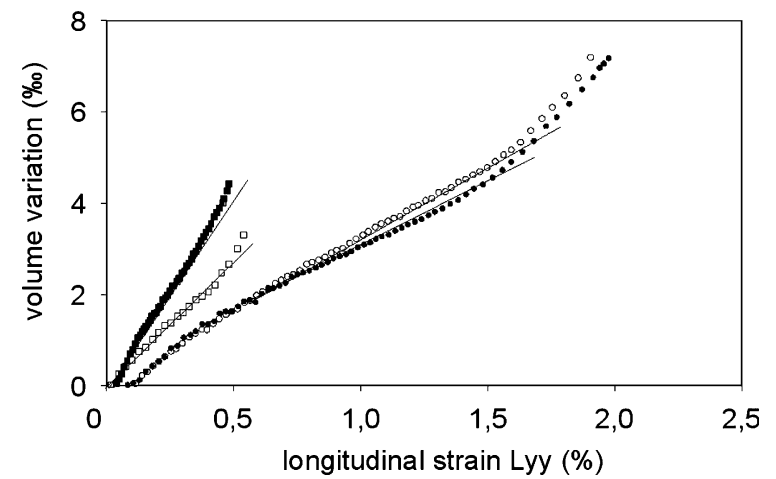

Fig. 15. Evolution of the theoretically elastic (full line) and experimental (symbols) volume variations during the tensile test for different aged samples: $(\bigcirc)$ unaged, $(\bullet) 6 \mathrm{~h}$ aged, $(\square) 54 \mathrm{~h}$ aged, and (ם) $120 \mathrm{~h}$ aged.

the experimental volume variation appears for lower longitudinal strain around $4 \times 10^{-3}$. This phenomenon relates the presence of an intrinsic damage of the hydrolysed material attributed to interfacial debonding.

3.2.4.2. Study of the local behaviour. A statistical analysis of the local strains was performed thanks to the mesh defined in Table 1. The extensometric gauge, being smaller than the sample thickness, the hypothesis of transverse isotropy is not confirmed. Then, the study of the surface variation (Eqs. (6) and (7)) is more appropriate:

$\frac{\Delta S}{S_{0}} \approx L_{x x}+L_{y y}$

$\left(\frac{\Delta S}{S_{0}}\right)_{\exp } \approx L_{x x}+L_{y y}$

The image correlation treatment allows evaluating the standard deviation evolution of the surface strain. This local approach provides a representative parameter of the surface strain heterogeneity. Since the difference between the strain levels observed for different ageing times are very important, another parameter has been introduced; the coefficient of variation, which is a normalized expression of the surface standard deviation. This one is depicted in Fig. 16 for different ageing times. The unaged sample is characterized by the highest value of the coefficient of variation whatever the longitudinal strain. This means that this unaged sample presents the most important strain activity which was attributed to relaxation phenomena of the internal stresses around the fibres. For aged sample, this coefficient is lower whatever the longitudinal strain. A more homogeneous mechanical behaviour and therefore

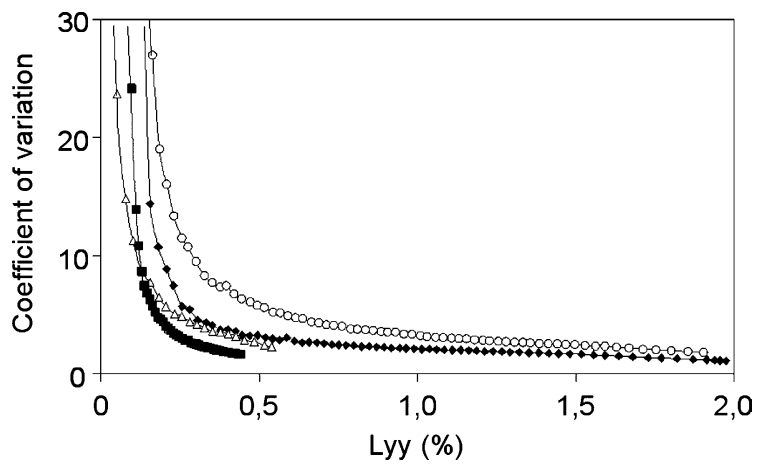

Fig. 16. Evolution of the surface variation standard deviation during the tensile test for different aged samples: $(\boldsymbol{\square})$ unaged, $(\triangle) 6 \mathrm{~h}$ aged, $(\diamond) 54 \mathrm{~h}$ aged, and (O) $120 \mathrm{~h}$ aged.

without strain localization ability is observed for these materials. It can be supposed that, in this case, the debonding at the interface is responsible for the lack of this strain accommodation ability.

\section{Conclusion}

In this work, a multiscale approach of the hygrothermal ageing of glass fibre reinforced PET composites has been developed in order to identify the various degradation mechanisms, to point out the correlations between them and to analyse their consequences on the macroscopic properties.

Under the present experimental conditions, hygrothermal ageing induces a rapid and drastic decrease of the mechanical strength of the composites. The water absorption is non-Fickian. This particular behaviour has been assigned to structural changes affecting the composite all along the ageing test. From the very beginning of ageing, the absorbed water induces not only a slight plasticisation of the matrix but, above all, hydrolysis of the polymer chains in the amorphous phase by a random scission mechanism. Hydrolysis generates oligomers that either diffuse slowly out of the material and solubilise in the ageing bath or crystallize in the interspherulitic zone. Therefore the production of oligomers modifies not only the hydrophilicity of PET but also its crystalline morphology, both factors being likely to interfere with the absorption process.

For very long ageing times, hydrolysis may affect even the crystalline zone of PET since the lamellar thickness decreases. Besides, interfacial decohesion occurs leading to the formation of cracks or voids that induce a additional uptake of water and the final osmotic cracking responsible for the material fracture. The extent of damage can be assessed at a mesoscopic scale by following strain heterogeneity using photomechanics. 


\section{Acknowledgements}

The authors would like to thank Pr G. Vigier (INSA Lyon), Pr L. David (UCB Lyon 1) and Pr A. Périchaud (Université de Provence) for their contribution to this work.

\section{References}

[1] Yang DK, Koros WJ, Hopfenberg HB, Stannett VT. The effects of morphology and hygrothermal aging on water sorption and transport in Kapton ${ }^{\circledR}$ polyimide. J Appl Polym Sci 1986;31(6): 1619-29.

[2] Han M-H, Nairn JA. Hygrothermal aging of polyimide matrix composite laminates. Compos Part A - Appl S 2003;34(10): 979-86.

[3] Mohd Ishak ZA, Berry JP. Effect of moisture absorption on the dynamic mechanical properties of short carbon fiber reinforced nylon 6,6. Polym Compos 1994;15(3):223-30.

[4] Mohd Ishak ZA, Berry JP. Hygrothermal aging studies of short carbon fiber reinforced nylon 6,6. J Appl Polym Sci 1994;51(13): 2145-55.

[5] Pires I. Aging in antifreeze of glass fiber reinforced polyamide. $\mathrm{PhD}$ thesis, University of Montpellier 2; 2000.

[6] Czigány T, Mohd Ishak ZA, Heitz T, Karger-Kocsis J. Effects of hygrothermal aging on the fracture and failure behavior in short glass fiber-reinforced, toughened poly(butylene terephthalate) composites. Polym Compos 1996;17(6):900-9.

[7] Mohd Ishak ZA, Ariffin A, Senawi R. Effects of hygrothermal aging and a silane coupling agent on the tensile properties of injection molded short glass fiber reinforced poly(butylene terephthalate) composites. Eur Polym J 2001;37(8):1635-47.

[8] Bárány T, Karger-Kocsis J, Czigány T. Effect of hygrothermal aging on the essential work of fracture response of amorphous poly(ethylene terephthalate) sheets. Polym Degrad Stab 2003;82 (2):271-8.

[9] Mac Mahon W, Birsdall HA, Johnson GR, Camilli CT. Degradation studies of polyethylene terephthalate. J Chem Eng Data 1959;4(1):57-79.

[10] Ravens DAS, Ward IM. Chemical reactivity of polyethylene terephthalate. Trans Farday Soc 1961;57:150-9.

[11] Zimmerman H. Degradation and stabilisation of polyesters. In: Grassie N, editor. Developments in polymer degradation -5 . London: Applied Science; 1979. p. 79.

[12] Golike RC, Lasoski Jr SW. Kinetics of hydrolysis of polyethylene terephthalate films. J Phys Chem 1960;64:895-8.

[13] Ballara A, Verdu J. Physical aspects of the hydrolysis of polyethylene terephthalate. Polym Degrad Stab 1989;26:361-74.
[14] Launay A, Thominette F, Verdu J. Hydrolysis of poly(ethylene terephthalate): a kinetic study. Polym Degrad Stab 1994;46: 319-24.

[15] Adamczak L. Study of hygrothermal ageing of three glass fibre reinforced thermoplastic composites. PhD thesis, Ecole Nationale Supérieure des Mines de Paris; 1995.

[16] Launay A, Thominette F, Verdu J. Hydrolysis of poly(ethylene terephthalate). A steric exclusion chromatography study. Polym Degrad Stab 1999;63:385-9.

[17] Gautier L, Mortaigne B, Bellenger V, Verdu J. Osmotic cracking nucleation in hydrothermal-aged polyester matrix. Polymer 2000;41:2481-90.

[18] Gautier L, Mortaigne B, Bellenger V. Interface damage study of hydrothermally aged glass-fibre-reinforced polyester composites. Compos Sci Technol 1999;59:2329-37.

[19] Pauchard V, Grosjean F, Campion-Boulharts H, Chateauminois A. Application of a stress-corrosion-cracking model to an analysis of the durability of glass/epoxy composites in wet environements Compos Sci Technol 2002;62:493-8.

[20] Oliveira BF, Creus GJ. An analytical-numerical framework for the study of ageing in fibre reinforced polymer composites. Compos Struct 2004;65:443-57.

[21] Vauthier E, Abry JC, Baillez T, Chateauminois A. Interactions between hygrothermal ageing and fatigue damage in unidirectionnal glass/epoxy composites. Compos Sci Technol 1998;58:687-92.

[22] Liao K, Tan YM. Influence of moisture induced stress on in situ fiber strength degradation of unidirectional polymer composites. Compos Part B - Eng 2001;32:365-70.

[23] Brinson LC, Gates TS. Viscoelasticity and aging of polymer matrix composites. In: Comprehensive composite materials. p. 333-68 [chapter 2.1].

[24] Pohl HA. Determination of carboxyl end groups in a polyester, polyethylene terephthalate. Anal Chem 1954;26(10):1614-6.

[25] Bergeret A, Pires I, Foulc MP, Abadie B, Ferry L, Crespy A. The hygrothermal behaviour of glass-fibre-reinforcedthermoplastic composites: a prediction of the composite lifetime. Polym Test 2001;20:753-63.

[26] Carter HG, Kibler KG. Langmuir-type model for anomalous moisture diffusion in composite resins. J Compos Mater 1978;12: $118-31$.

[27] Staggs JEJ. Modelling random scission of linear polymers. Polym Degrad Stab 2002;76:37-44.

[28] Kostoglou M. Mathematical analysis of polymer degradation with chain-end-scission. Chem Eng Sci 2000;16(3):2507-13.

[29] Chaupart N, Serpe G, Verdu J. Molecular weight distribution and mass changes during polyamide hydrolysis. Polymer 1998;39(6-7):1375-80.

[30] King E, Robinson S, Cameron RE. Effect of hydrolytic degradation on the microstructure of quenched, amorphous poly(glycolic acid): an X-ray scattering study of hydrated samples. Polym Int 1999;48:915-20. 